\title{
Stochastic Model for Surface Erosion Via Ion-Sputtering: Dynamical Evolution from Ripple Morphology to Rough Morphology
}

\author{
Rodolfo Cuerno ${ }^{1}$, Hernán A. Makse ${ }^{1}$, Silvina Tomassone ${ }^{2}$, Stephen T. Harrington ${ }^{1}$, and H. Eugene Stanley ${ }^{1}$ \\ ${ }^{1}$ Center for Polymer Studies and Physics Department, Boston University, Boston, MA 02215 \\ ${ }^{2}$ Physics Department, Northeastern University, Boston, MA 02115
}

\begin{abstract}
Surfaces eroded by ion-sputtering are sometimes observed to develop morphologies which are either ripple (periodic), or rough (non-periodic). We introduce a discrete stochastic model that allows us to interpret these experimental observations within a unified framework. We find that a periodic ripple morphology characterizes the initial stages of the evolution, whereas the surface displays self-affine scaling in the later time regime. Further, we argue that the stochastic continuum equation describing the surface height is a noisy version of the Kuramoto-Sivashinsky equation.
\end{abstract}

PACS numbers: 64.60.Ht, 79.20.Rf, 68.35.Rh

A remarkable feature of erosion processes via ion sputtering in amorphous materials is the formation of a pattern consisting of a ripple structure, aligned in directions either parallel to or perpendicular to that of the bombarding beam of ions [1,2]. Indeed, we might expect that erosion tends to erase every possible feature of the surface morphology, and that the presence of noise in the system would further act against the formation of such a periodic pattern. Only recently have there been experimental [3] and theoretical [4] attempts to understand the formation of a ripple structure in the more general context of non-equilibrium interface growth phenomena [3]. Many such interfaces are "rough" and exhibit self-affine scaling at long distances and long times [1]; experimentally, one finds that surfaces eroded by ion bombardment also exhibit self-affine scaling behavior [5]. An outstanding question is then how to reconcile these observations with the formation of the periodic ripple structure.

In this Letter, we introduce a discrete stochastic model that incorporates the main physical mechanisms believed to influence the dynamics of the eroded surface morphology. We show that this model is characterized by an initial stage in which the surface morphology displays a ripple structure, and that subsequent stages are characterized by a crossover to a rough surface in the universality class of the Kardar-Parisi-Zhang (KPZ) equation [6]. We argue that the stochastic equation which provides the continuum description of our model is a noisy version [7] of the Kuramoto-Sivashinsky (KS) equation [8.97. Thus we interpret the formation of a periodic pattern [3] and the development of rough interfaces [5] in the ion-sputtered systems as the early and late regimes respectively of the same dynamical process.

We first consider the main mechanisms 10] that determine the surface morphology undergoing ion bombardment.

(i) Erosion - In ion sputtering, an initially flat substrate is bombarded with a well-collimated beam of heavy ions carrying a certain kinetic energy, and forming a precise angle with the normal to the uneroded surface. The phenomena leading to erosion take place within some fi- nite distance from the surface. Namely, the ions penetrate inside the solid and induce along their path cascades of collisions among the atoms of the substrate. Atoms located at the surface may be affected by these collisions and acquire enough energy to leave the surface 11. Consequently, atoms located at the bottom of troughs gain more energy on average and they are preferentially eroded as compared to those on the peaks of crests [12]. This "instability" can be thought of as a negative surface tension, since the surface tends to maximize its area.

(ii) Surface Diffusion - In physical systems there is a stabilizing mechanism that balances the negative surface tension, surface diffusion, which is always present at a non-zero temperature. Particles on the surface tend to diffuse looking for highly coordinated positions, a relevant phenomenon for sputtering [3:5] as well as for molecular-beam epitaxy (MBE) 13.

When the angle between the local normal to the surface and the incident beam approaches the grazing value, there is an increase in the reflection of the ions by the surface and the rate of erosion diminishes. This surface effect is beyond the approximations made in Sigmund's theory of ion sputtering [11], and is reflected in the angle dependence of the sputtering yield, $Y(\varphi)$, defined to be the number of eroded particles divided by the total number of bombarding ions. Here $\varphi$ is the local angle of the ion trajectories to the surface normal at each point [14]. Typically [2], $Y(\varphi)$ is symmetric around $\varphi=0$, presents a maximum between $60^{\circ}$ and $80^{\circ}$, and decreases to zero as $\varphi \rightarrow 90^{\circ}$.

To define the model, we introduce two dynamical rules, one to account for erosion and one to account for surface diffusion. The rule for erosion incorporates the unstable behavior described above as well as the phenomenological dependence of the sputtering yield as a function of $\varphi$. The model for the case of $1+1$ dimensions [15] is defined on a square lattice of lateral size $L$, with periodic boundary conditions in the horizontal direction. The initial interface is a horizontal line separating occupied sites (below) from empty sites (above). We choose randomly a 
site $i$ at the interface where $i=1, \ldots, L$. The chosen site is subject to erosion with probability $p$, or to diffusion with probability $1-p$, where the rules are as follows:

(i) Erosion (probability $p$ ) - 16] We compute $\varphi \equiv$ $\tan ^{-1}\left[\left(h_{i+1}-h_{i-1}\right) / 2\right]$, where $h_{i}$ is the height of the interface at site $i$, and apply the erosion rule with probability $Y(\varphi)$, as given in Fig. $1 a$. To erode, we count the number of occupied neighbors inside a square box of size $3 \times 3$ lattice spacings centered in the chosen site $i$ (box rule). We empty the site with an erosion probability $p_{e}$ proportional to the number of occupied cells in the box (see Fig. 1b). Thus the box rule favors the erosion of troughs as compared to the peaks of crests, and therefore is the source of the instability in the ion-sputtered system.

(ii) Surface Diffusion (probability $1-p)-\mathrm{A}$ diffusive move of the particle $i$ to a nearest neighbor column is attempted with hopping probability $w_{i \rightarrow f} \equiv$ $\left[1+\exp \left(\Delta \mathcal{H}_{i \rightarrow f} / k_{B} T\right)\right]^{-1}$, where $\Delta \mathcal{H}_{i \rightarrow f}$ is the energy difference between the final and initial states of the move. Following [17], we choose $\mathcal{H} \equiv(J / 2) \sum_{\langle i, j\rangle}\left(h_{i}-h_{j}\right)^{2}$.

A continuum equation that describes the dynamics of the interface height has the form

$$
\partial_{t} h(\boldsymbol{x}, t)=\nu \nabla^{2} h-\kappa \nabla^{4} h+\eta(\boldsymbol{x}, t)+f_{Y}[h(\boldsymbol{x}, t)] .
$$

Here $h(\boldsymbol{x}, t)$ is the height of the interface at position $\boldsymbol{x}$ and time $t, \nu$ is a negative surface tension coefficient, $\kappa$ is a positive coefficient that accounts for the surface diffusion, and $\eta(\boldsymbol{x}, t)$ is a Gaussian noise term with short range correlations and strength $2 D$, that accounts for the fluctuations in the flux of incoming particles. The functional $f_{Y}[h]$ takes into account the contribution of nonlinear terms, which appear in the equation of motion due to the effect of $Y(\varphi)$, itself a nonlinear function of the local slope $\nabla h \equiv \tan \varphi$. The nonlinearities become more relevant in the equation of motion at the late regime of the evolution when large slopes develop, see below.

First we study the model in the case $Y(\varphi) \equiv 1$, which approximately holds at the early stages of evolution. We focus on the time dependence of the total interface width $W(t) \equiv\left\langle L^{-1} \sum_{i=1}^{L}\left(h_{i}(t)-\overline{h(t)}\right)^{2}\right\rangle^{1 / 2}$, where $\overline{h(t)} \equiv L^{-1} \sum_{i=1}^{L} h_{i}(t)$, and the brackets denote an average over realizations of the noise. The erosion rule alone - corresponding to $p=1, Y(\varphi) \equiv 1$-leads to $W(t) \sim t$, which can be attributed to $\nu$ in (11) being a negative number [18]. Moreover, by considering only the erosion rule, but defined with probability $1-p_{e}$, we find that the interface has the scaling properties of the Edwards-Wilkinson (EW) equation [19] that is obtained from (11) with $\nu>0, \kappa=0$, and $f_{Y}[h]=0$. We understand this result since one favors the erosion of peaks as compared to valleys, leading to the smoothing mechanism characteristic of a positive surface tension. On the other hand, the surface diffusion mechanism alone (for $p=0, Y(\varphi) \equiv 1)$ is well described by (11) with $\nu=0$, $\kappa>0$, and $f_{Y}[h]=0$, i.e., the linear MBE equation [17, 13. When mechanisms (i) and (ii) above are considered simultaneously for $J / k_{B} T=5, p=0.5$, and
$Y(\varphi) \equiv 1$, we obtain the different stages of the time evolution displayed in Fig. $2 a$ 21]. There exists a first region 22 for which $W(t) \sim t^{\beta_{1}}$, with $\beta_{1}=0.38 \pm 0.02$, the growth exponent for the linear MBE universality class, after which $W(t)$ scales with $\beta_{2}>0.5$ due to the instability caused by $\nu<0$. In this case, a linear stability analysis of (1) shows that there is a maximally unstable mode in the system, $k_{m}=(|\nu| / 2 \kappa)^{1 / 2}$, and therefore the surface is almost periodic (inset in Fig. $2 a$ ).

Next we consider the model with $Y(\varphi)$ shown in Fig. 1a. The results are not expected to depend strongly on the specific form of $Y(\varphi)$, so long as it preserves the existence of a maximum, and $Y(0) \neq 0, Y\left(90^{\circ}\right)=0$ [23]. Fig. 3 shows the structure factor $S(\boldsymbol{k}) \equiv\langle\widehat{h}(\boldsymbol{k}, t) \widehat{h}(-\boldsymbol{k}, t)\rangle$ at the onset of the instability. Here $\widehat{h}(\boldsymbol{k}, t)$ is the Fourier transform of $h_{i}(t)-\overline{h(t)}$. As we see, the early stages of the dynamics are still dominated by the periodic ripple structure defined by the competition between surface tension and surface diffusion, described by the linear part of (11).

For later times, the large slopes built up by the instability induce nonlinear effects, and the interface results in a rough morphology (inset of Fig. 2b). In Fig. $2 b$, we present the time evolution of $W(t)$ for the complete model. We again observe a first regime [22] with $\beta_{1}=0.38 \pm 0.03$, followed by unstable erosion $\left(\beta_{2}>0.5\right)$. For later stages, we find $\beta_{3}=0.23 \pm 0.03$, consistent with EW, after which a crossover to $\beta_{4}=0.28 \pm 0.03$ is found. Finally, the width saturates due to the finite size of the system. Note that the value of the growth exponent for the $\mathrm{KPZ}$ equation is $\beta_{\mathrm{KPZ}}=1 / 3$ [6]. At saturation, $S(k)$ displays the small momenta behavior $S(k, t) \sim k^{-2}$, consistent with the scaling of both the EW and KPZ universality classes (see Fig. 3). To determine if a KPZ nonlinearity is present in Eq. (1), we compute the mean velocity $v(m)$ of the interface in the saturated regime as a function of an average tilt $m \equiv\langle\nabla h\rangle$ imposed by using helical boundary conditions. If we assume that the relevant nonlinearity in (11) is of the KPZ type, then $f_{Y}[h]=(\lambda / 2)(\nabla h)^{2}$. Taking spatial and noise averages in (11), $v=v_{0}+(\lambda / 2) m^{2}$, where $v_{0}$ is the velocity of the untilted interface 24]. The parabolic shape of $v(m)$ obtained in our simulations (see Fig. 4) leads to the conclusion that the long time and long distance behavior of the model falls into the KPZ universality class. Moreover, the continuum equation describing the model ion-sputtered surfaces is the noisy KS equation

$$
\partial_{t} h=\nu \nabla^{2} h-\kappa \nabla^{4} h+\frac{\lambda}{2}(\nabla h)^{2}+\eta(\boldsymbol{x}, t) .
$$

To compare the dynamics of (2) with those obtained for the discrete model, we integrate numerically Eq. (2) in $1+1$ dimensions. Fig. 5 shows the behavior of the function $W(t)$. We observe the same crossovers as in the model 22]: $\beta_{1}=0.38 \pm 0.03$ corresponding to the linear $\mathrm{MBE}$ case, followed by unstable growth $\beta_{2}>0.5$. Then, a transition to EW behavior $\beta_{3}=0.25 \pm 0.03$ 
is observed, after which the nonlinearities dominate and a KPZ growth with $\beta_{4}=0.30 \pm 0.03$ is obtained. Finally, the interface width saturates due to the finite size of the system. Consistent with these numerical findings, the late scaling of Eq. (2) has been shown through a renormalization-group calculation [7] to be that of the KPZ equation in $1+1$ and $2+1$ dimensions. As we see, both in the model and the noisy KS, there is a long crossover time from $\mathrm{EW}$ to $\mathrm{KPZ}$ behavior, responsible for the difference between $\beta_{4}$ and $\beta_{K P Z}$, and for the narrow window in which $\beta_{4}$ is observed - we find that the width of this window increases systematically with $L$. A similar phenomenon is well known to occur in the deterministic KS equation in $1+1$ dimensions, see Sneppen et al. in [9].

Finally, we compare the results of the model with observations of recent experiments 25]. The experimental development of a ripple structure [3] is well understood in terms of the unstable linear theory of ion-sputtering describing the early stages of the time evolution of the model presented here. Moreover, the model predicts that in the late regime the large slopes generated by the unstable growth trigger the action of nonlinearities which stabilize the surface. The nonlinearity we find is of the KPZ type, consistent with the experimental observation of KPZ scaling reported by Eklund et al. |5|. To confirm the above picture, it would be of interest to study experimentally if both regimes do effectively take place in the time evolution of the same physical system.

We acknowledge discussions with A. L. Barabási, G. Carter, S. Havlin, and K. B. Lauritsen. R. C. acknowledges support from Ministerio de Educación y Ciencia, Spain. The Center for Polymer Studies is funded by NSF.

[1] P. Meakin, Phys. Rep. 235, 189 (1993); T. Halpin-Healey and Y.-C. Zhang, ibid. 254, 215 (1995); A.-L. Barabási and H. E. Stanley, Fractal Concepts in Surface Growth (Cambridge University Press, Cambridge 1995)

[2] G. Carter et al., in Sputtering by Particle Bombardment, Vol. II, edited by R. Behrisch, (Springer-Verlag, Heidelberg 1983), p. 231.

[3] E. Chason et al., Phys. Rev. Lett. 72, 3040 (1994); T. M. Mayer et al., J. Appl. Phys. 76, 1633 (1994).

[4] R. Cuerno and A. L. Barabási, Phys. Rev. Lett. 74, 4746 (1995).

[5] E. A. Eklund et al., Phys. Rev. Lett. 67, 1759 (1991); E. A. Eklund et al., Surf. Sci. 285, 157 (1993); J. Krim et al., Phys. Rev. Lett. 70, 57 (1993); H.-N. Yang, G.-C. Wang, and T.-M. Lu, Phys. Rev. B 50, 7635 (1994).

[6] M. Kardar, G. Parisi and Y.-C. Zhang, Phys. Rev. Lett. 56, 889 (1986).

[7] R. Cuerno and K. Lauritsen, Phys. Rev. E 52, 4853 (1995). For $d>2$, see L. Golubović and R. Bruinsma,
Phys. Rev. Lett. 66, 321 (1991); ibid. 67, 2747 (E) (1991).

[8] Y. Kuramoto and T. Tsuzuki, Prog. Theor. Phys. 55, 356 (1977); G. I. Sivashinsky, Acta Astronaut. 6, 569 (1979).

[9] S. Zaleski, Physica D 34, 427 (1989); K. Sneppen et al. Phys. Rev. A 46, R7351 (1992); F. Hayot et al., Phys. Rev. E 47, 911 (1993).

[10] Here we neglect redeposition of the eroded material, as well as shadowing effects among different surface features. These assumptions have been shown [3] to hold at the initial stages of surface evolution. The roughening taking place in the model at late time stages is again consistent with them.

[11] P. Sigmund, Phys. Rev. 184, 383 (1969).

[12] P. Sigmund, J. Mat. Sci. 8, 1545 (1973); R. M. Bradley and J. M. E. Harper, J. Vac. Sci. Technol. A 6, 2390 (1988).

[13] C. Herring, J. Appl. Phys. 21, 301 (1950); W. W. Mullins, J. Appl. Phys. 28, 333 (1957); in the context of stochastic models, see D. E. Wolf and J. Villain, Europhys. Lett. 13, 389 (1990); S. Das Sarma and P. I. Tamborenea, Phys. Rev. Lett. 66, 325 (1991).

[14] When we fix the type of bombarding ion, the material of the substrate, and the energy of the incoming beam, $Y(\varphi)$ is a function of $\varphi$ only.

[15] The generalization to $2+1$ dimensions will be reported elsewhere.

[16] We consider normal incidence of the ions onto the substrate.

[17] M. Siegert and M. Plischke, Phys. Rev. E 50, 917 (1994).

[18] Strictly speaking, $\nu<0$ implies exponential growth for $W$, which can not be attained in the present definition of the box rule, since at most only $L$ particles can be eroded per unit time, leading to $W \sim t$ as the fastest growth for the width.

[19] S. F. Edwards and D. R. Wilkinson, Proc. R. Soc. Lond. A 381, 17 (1982).

[20] K. Lauritsen (private communication).

[21] Qualitatively the results do not change when other values of $J / k_{B} T$ and $p$ are considered.

[22] An initial random erosion regime $\left(\beta_{0}=0.5\right)$ is also observed before correlations build up in the system.

[23] G. Carter et al., Surf. Interface Anal. 20, 90 (1993); A. N. Protsenko, Nucl. Instr. and Meth. B 82, 417 (1993).

[24] J. Krug and H. Spohn, Phys. Rev. Lett. 64, 2332 (1990).

[25] Based on our identification of Eq. (2) as describing the model, and the scaling of this equation in $2+1$ dimensions predicted in [7], we expect our results to carry over for the physical case of two-dimensional surfaces. An important ingredient in $2+1$ dimensions is the anisotropy between the two substrate directions induced by the ion beam. This leads to effects such as the change in the orientation of the ripples as a function of the angle of incidence [12], and could be accounted for by introducing a non-zero angle of incidence. 
FIG. 1. (a) Sputtering yield $Y(\varphi)$ as a function of the angle $\varphi$. (b) Box rule for erosion. We define $p_{e}$ as the number of occupied neighboring sites (grey squares) inside the $3 \times 3$ box centered at site $i$ (black square), normalized by 7 . The examples shown correspond to $(i) p_{e}=1$ and $(i i) p_{e}=3 / 7$.

FIG. 2. (a) Time evolution of the surface width for the cases $Y(\varphi) \equiv 1$ and $L=50$. The solid line is the consecutive slope of the width, showing the value of the growth exponent $\beta$ in each regime. The inset shows the ripple structure of the interface at $t=1000$. The saturation observed in $W(t)$ is due to the discreteness of the lattice: the erosion rule breaks down when the local slopes of the interface are bigger than 3 [20]. This effect can be avoided by using a bigger box. (b) Interface width as a function of time for the full model showing the regimes of the evolution for $L=2048$. As in (a), the solid line is the consecutive slope. The inset shows a portion of the rough morphology at the late regime, where the self-affine scaling behavior holds. The arrows indicate the times at which the structure factor is displayed in Fig. 3.
FIG. 3. Structure factor computed using the full model for a system with $L=2048$. For $t=300$, averaged over 2600 noise realizations $(\diamond)$, and for $t=1.7 \times 10^{6}$, averaged over 39 realizations $(\bullet)$ (see arrows in Fig. $2 b$ ). The solid line is a fit to the exact solution of the discretized linear part of Eq. (1). The dashed straight line has slope -2 .

FIG. 4. Plot of the mean velocity $v(m)$ as a function of the average tilt $m$ of the interface, calculated in the saturated regime for $L=128$, and averaged over 810 noise realizations. The solid line is a fit to a parabola.

FIG. 5. Log-log plot of the interface width as a function of time obtained in the numerical integration of Eq. (2). The mesh is $\Delta x=1.0$ and the time step $\Delta t=0.005$. Parameter values are $\nu=-1, \kappa=1, \lambda=4, D=0.5$, and $L=1024$. The solid line gives the consecutive slopes. 

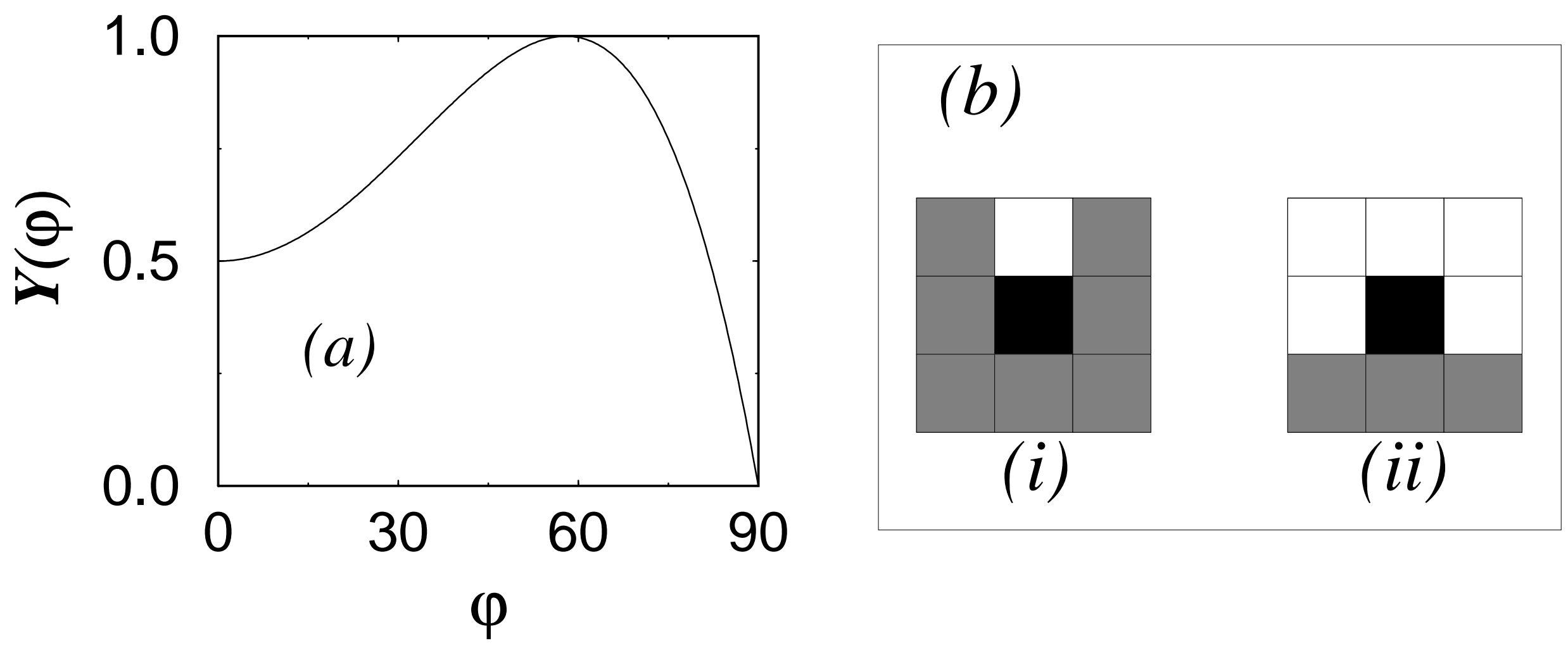


$$
\text { 䒠 }
$$




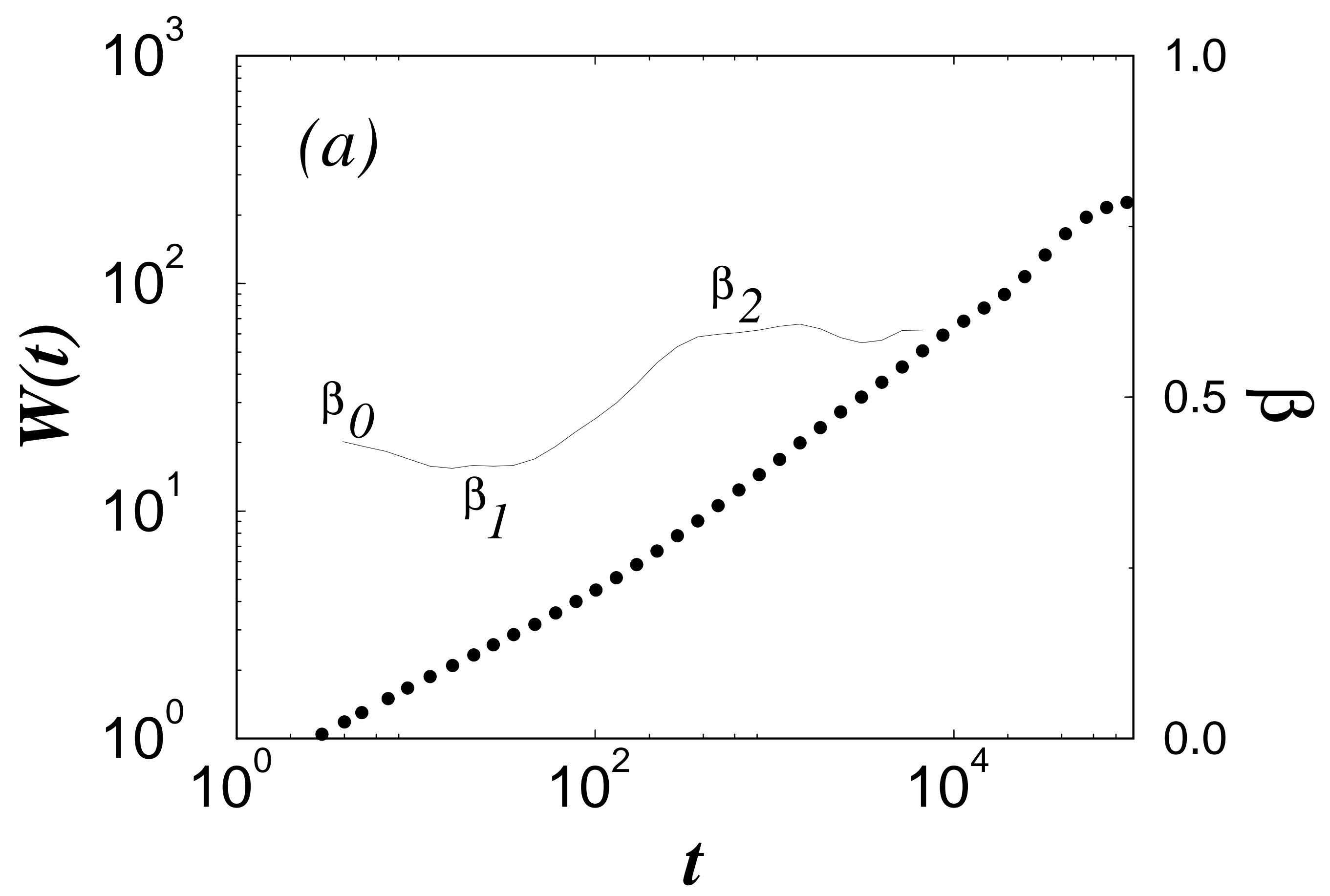




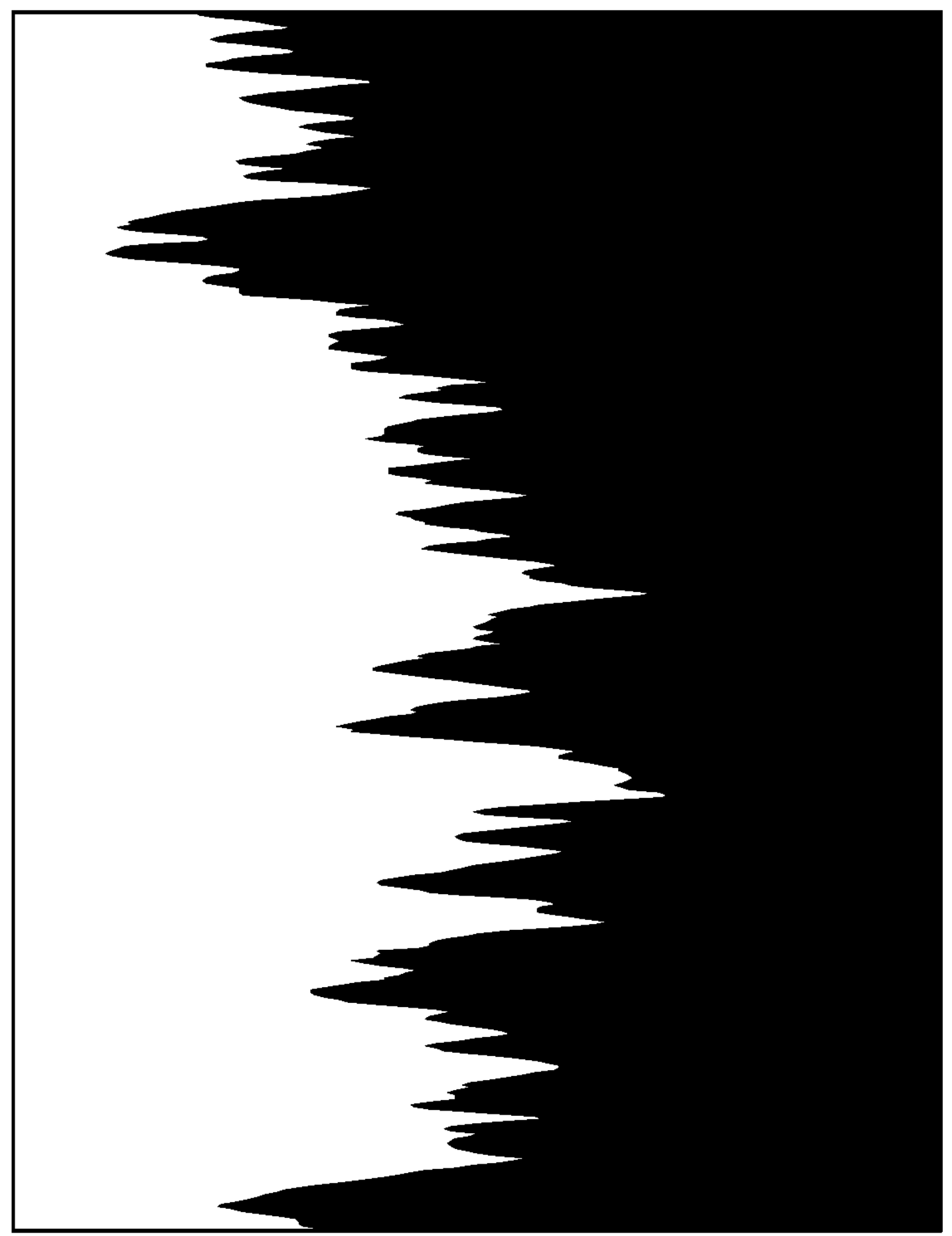




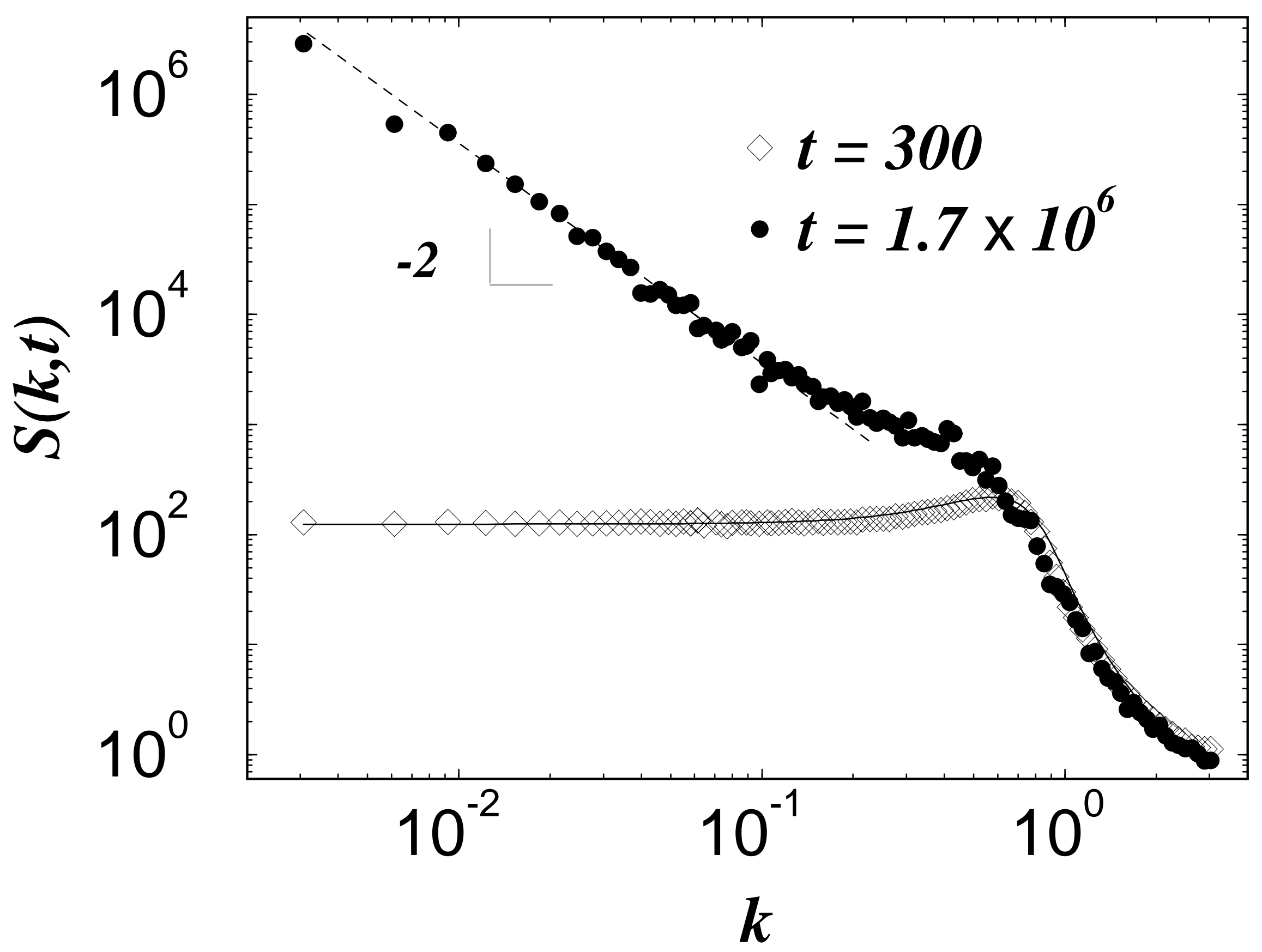




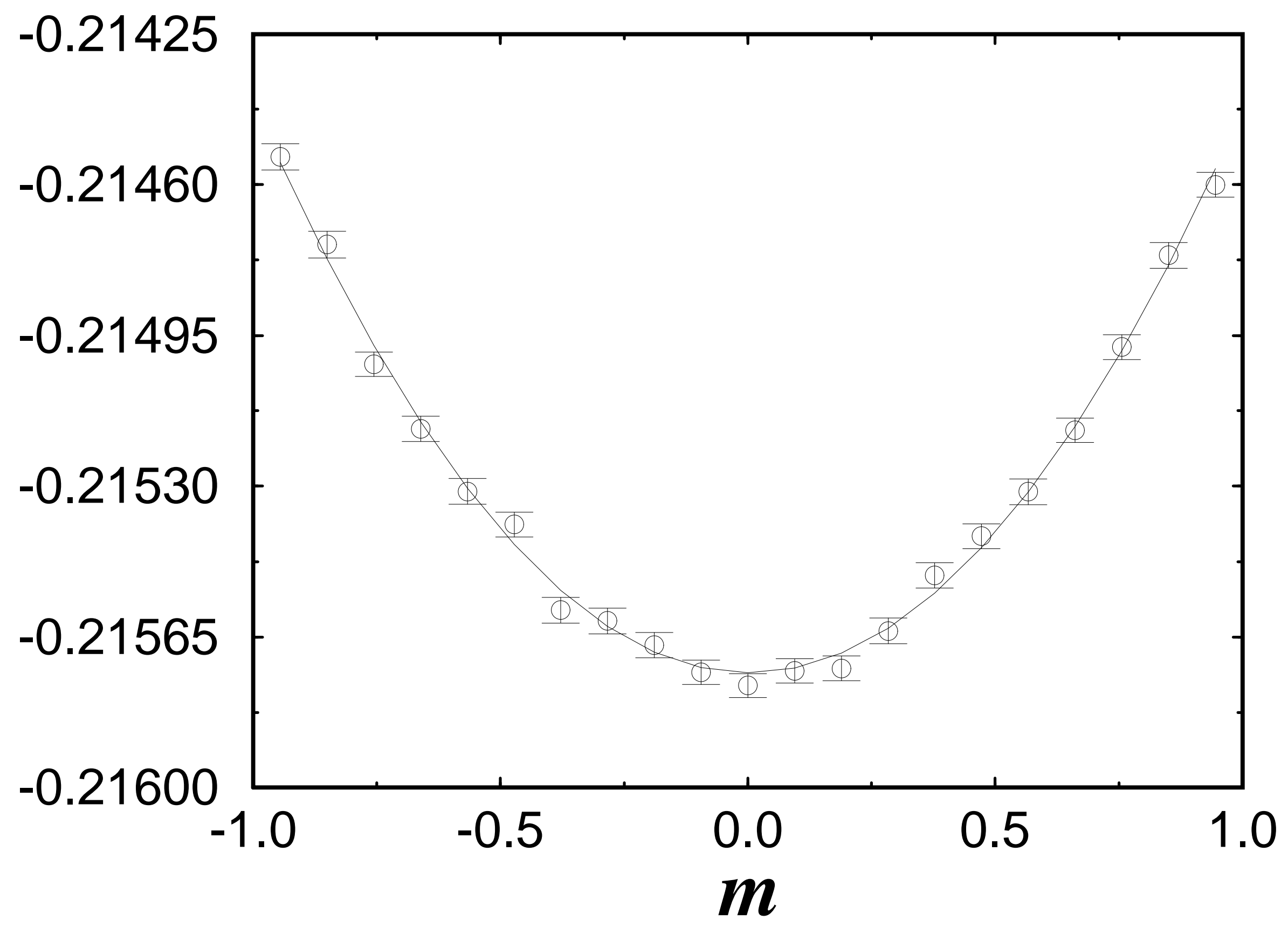




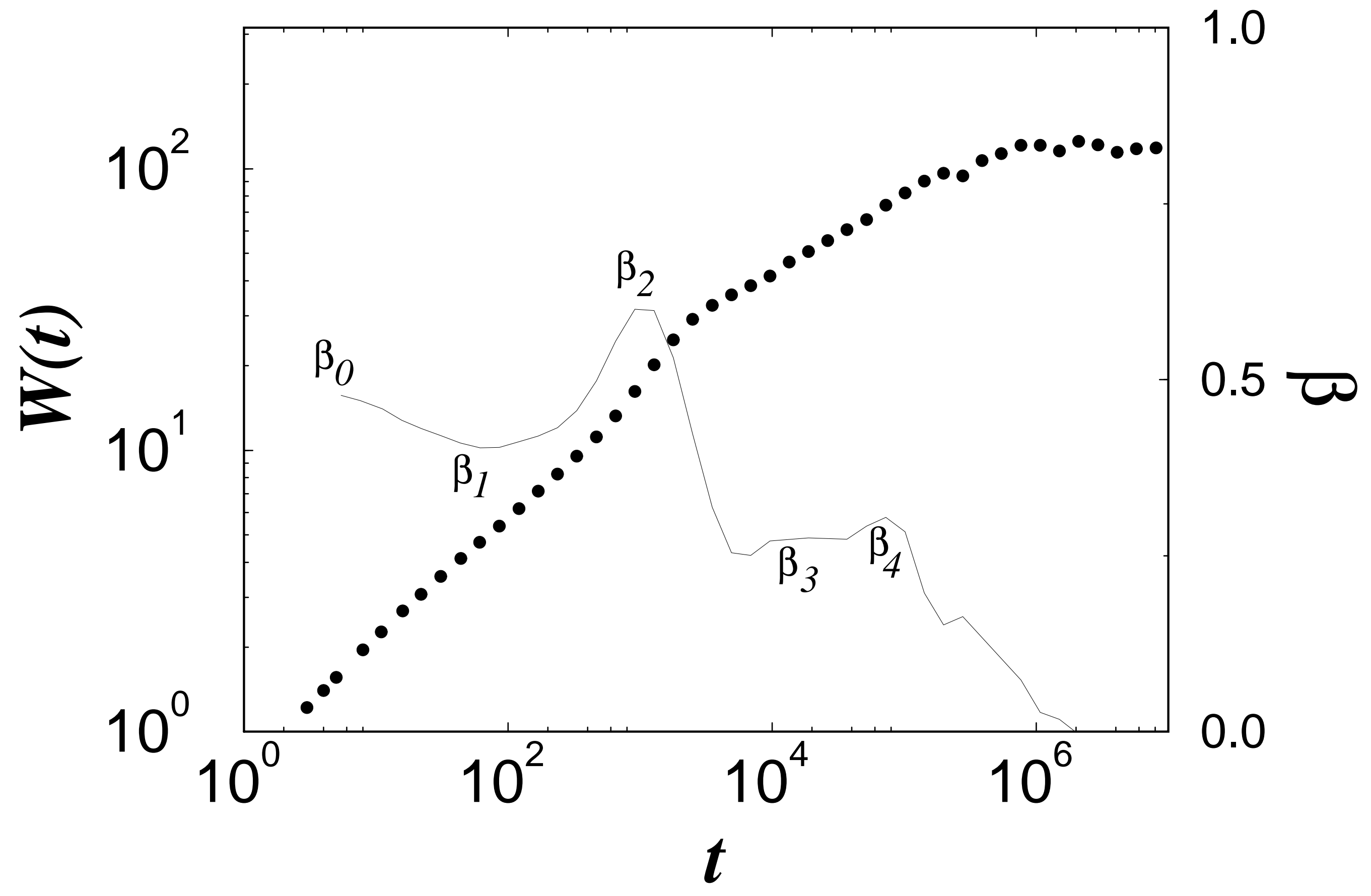

